\title{
Subchondral Insufficiency Fracture in the Lateral Compartment of the Knee in a 64-Year-Old Marathon Runner
}

\author{
Vincent VG An, BSc ${ }^{1}$, Mathias van den Broek, $\mathrm{MD}^{2}$, and Sam Oussedik, FRCS ${ }^{2}$ \\ ${ }^{1}$ Faculty of Medicine, University of Sydney, Sydney, Australia; ${ }^{2}$ Department of Orthopaedics, University College London Hospitals NHS Trust, London, UK
}

A 64-year-old marathon runner presented to our clinic with a history of worsening pain in her left knee. Following magnetic resonance imaging, the diagnosis of subchondral insufficiency fracture of the knee (SIFK) was made. A course of conservative management via bisphosphonates was initiated. The patient successfully recovered and returned to full athletic function, having recently returned to competitive marathon running. This article aims to present this unusual case of lateral compartment SIFK and summarise the literature on this uncommon condition.

Keywords: Knee, Femur, Fracture, Insufficiency, Runner

Subchondral insufficiency fractures (SIF) are a type of stress fracture which occurs below the cartilage on the weight bearing surface of a bone. SIF occur when normal physiological forces are repeatedly applied to an area of bone compromised by nontumorous disease, resulting in fracture.

Subchondral insufficiency fractures of the knee (SIFK) are closely linked with spontaneous osteonecrosis of the knee (SONK) and are thought to be the inciting event which leads to $S O N K^{1)}$. Due to this close relationship, SIFK and SONK are sometimes used interchangeably as terms, although some authors have suggested SONK should be renamed as $\mathrm{SIFK}^{2,3)}$. Both present with acute knee pain in the absence of trauma, which manifests radiologically as bone marrow oedema surrounding a necrotic area if progression to SONK has occurred. These lesions most com-

Received July 7, 2017; Revised (1st) August 11, 2017;

(2nd) September 11, 2017; Accepted September 26, 2017

Correspondence to: Vincent VG An, BSc

Faculty of Medicine, University of Sydney, Camperdown, Sydney, NSW

2135, Australia

Tel: +61-405-648-186, Fax: +61-2-9746-6686

E-mail: vian2424@uni.sydney.edu.au

This is an Open Access article distributed under the terms of the Creative Commons Attribution Non-Commercial License (http://creativecommons.org/licenses/by-nc/4.0/) which permits unrestricted non-commercial use, distribution, and reproduction in any medium, provided the original work is properly cited. monly occur in the weight bearing medial compartment of the $\mathrm{knee}^{4)}$.

This paper describes an unusual case of SIFK in the lateral femoral condyle of a female ultra-marathon athlete who was successfully managed with non-operative treatment.

\section{Case Report}

Written consent was obtained from the patient to present this case. A 64-year-old female ultra-marathon runner was referred to our clinic with a 5 month history of left lateral knee pain. The pain developed 50 weeks into a rigorous year-long marathon running campaign during which she completed 52 marathons in 52 weeks. Whilst she was able to complete all marathons, the pain became significant after her final marathon, forcing her to restrict herself to walking up until her presentation. She also reported several episodes of night pain in the left knee which woke her from sleep. She had no relevant medical history of note and family history was negative. On physical examination, her left knee exhibited a grade one effusion with tenderness on palpation of the lateral joint line. There was a normal range of motion and good stability of this left knee. On visual inspection, the left leg appeared to be in more valgus alignment than the right.

A plain radiograph revealed a subchondral lucency within the lateral femoral condyle, with normal appearance of the tibial sur- 
face (Fig. 1). Due to the retrospective nature of this case report, no long-leg anteroposterior radiograph was available. Additional magnetic resonance imaging (MRI) revealed a subchondral insufficiency fracture on the extensor surface of the lateral femoral condyle and the presence of a large amount of bone marrow oedema measuring $2.4 \mathrm{~cm}$ in the anteroposterior plane and $2.1 \mathrm{~cm}$ transversely (Fig. 2). Menisci, collateral and cruciate ligaments were intact. A bone density scan was undertaken which demonstrated osteopenia in the femoral neck (density, $0.917 \mathrm{~g} / \mathrm{cm}^{2} ; \mathrm{T}$ score, -1.1; Z-score, 0.4 ) and lumbar spine (density, $0.723 \mathrm{~g} / \mathrm{cm}^{2}$; T-score, -1.2 ; Z-score, 0.2 ) with a total femoral density of $0.853 \mathrm{~g} /$

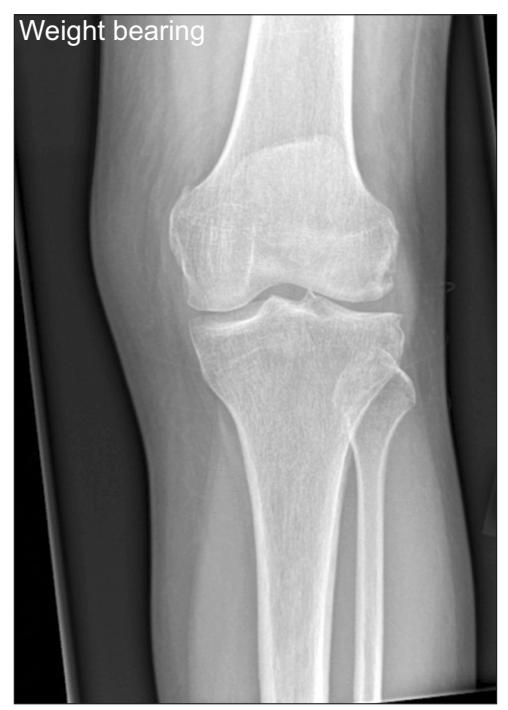

Fig. 1. Plain radiograph of the knee demonstrating subchondral lucency within the femoral condyle but otherwise normal appearance. $\mathrm{cm}^{2}$, T-score of -0.7 and Z-score of 0.3 .

Given the severity and restriction of life conferred by the symptoms, surgical management was discussed with the patient, including offloading, distal femoral osteotomy, resurfacing procedures, and partial joint replacement. However, the patient declined and instead agreed to start a course of bisphosphonates, consisting of three tri-monthly infusions of pamidronate with a view to switching to alendronic acid in the long term. A program of physiotherapy was also instituted, centred around non-weight bearing strengthening exercises of the leg but avoiding impact exercises. After her initial course of bisphosphonates (6 months after her initial presentation), she was able to cycle without any pain but was still unable to run. At this point, it was advised that impact exercises should continue to be avoided. MRI followup at this point revealed some improvement in both the volume and intensity of the bone marrow oedema in the lateral femoral condyle (Fig. 2). The patient was referred for a second course of bisphosphonate infusion treatment, and commenced this treatment at 12 months after her first presentation. At the 15-month mark following her second injection, MRI revealed marked improvement in bone marrow edema, and the decision was made to cease her bisphosphonate therapy.

Follow-up at 18 months showed complete resolution of her everyday symptoms. MRI was repeated and revealed a further reduction in her bone marrow oedema in the lateral femoral condyle. At the latest follow-up at 30 months, the patient reported no further knee pain and a successful return to ultra-marathon running.
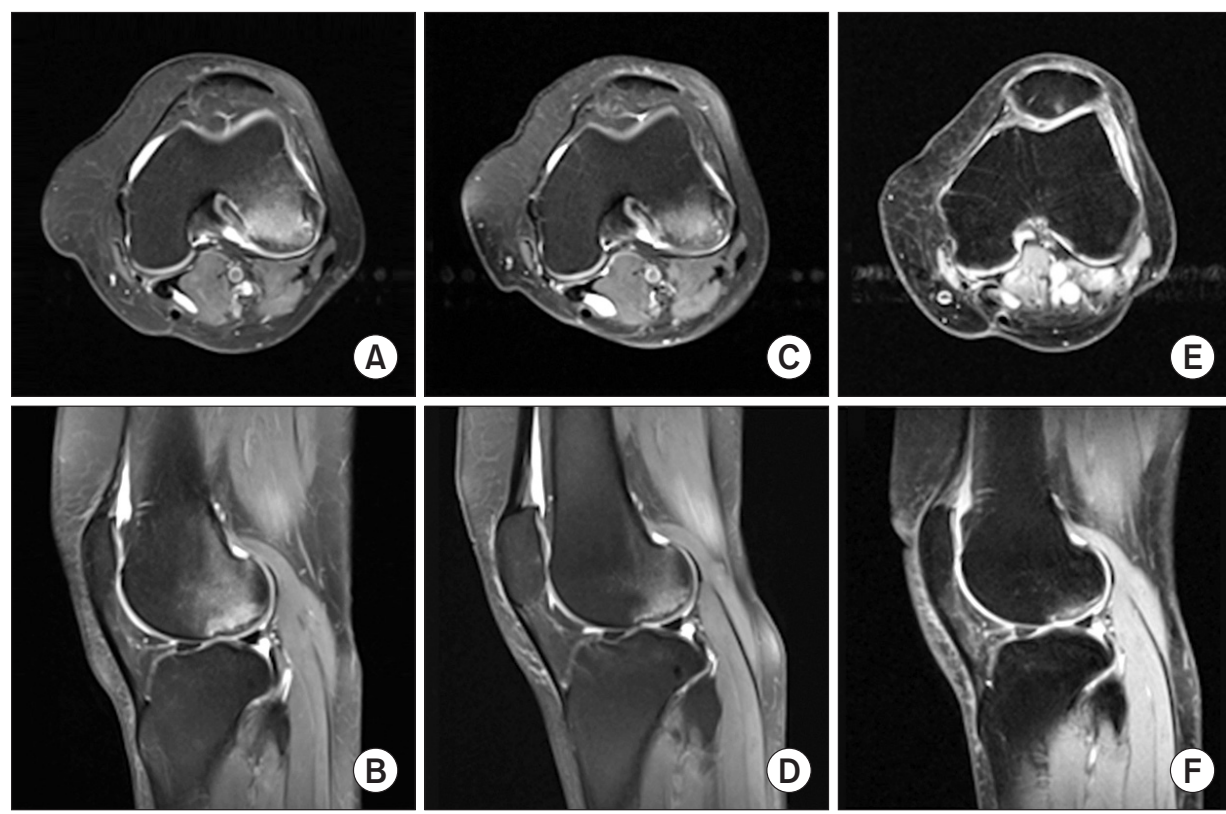

Fig. 2. Axial (top) and sagittal (bottom) magnetic resonance imaging scans demonstrating the progression of the patient's subchondral insufficiency fracture at presentation $(A, B)$ and at 6 months $(C, D)$ and 30 months of follow-up (E, F). Note the decreasing area of bone marrow oedema in the femoral condyle. There is significant articular oedema at 30 months as the image was taken on the day immediately after the patient had run a marathon. 


\section{Discussion}

SIF of the knee is a sparsely described and poorly understood condition, with most cases presenting in the medial weight bearing compartment of the knee. Cases of the lateral compartment, as presented in this case report, are even rarer.

Due to their similar presentation and imaging findings, the relationship and distinction between SIFK and SONK remains a topic of debate. Some authors consider SIFK and SONK to be synonymous or equivalent pathological processes ${ }^{2,5)}$, whilst others believe SIFK to be an inciting event for SONK to occur. The latter hypothesis is well-supported by histopathological findings which have correlated MRI findings of osteonecrosis or pre-osteonecrotic bone to microfractures or disruptions in the subchondral bony trabeculae $e^{4)}$.

The underlying cause of these subchondral fractures is also a topic of controversy. SIFK has been attributed to low bone mineral density (BMD) by Akamatsu et al. ${ }^{6}$, who found that female patients with SONK had lower BMD than a set of controls and thus hypothesized osteoporotic microfractures lead to the development of osteonecrosis. A more recent study by Nelson et al. ${ }^{7)}$ (2014) examined a population of patients with radiologically diagnosed SIFK, and found that only $16 \%$ of patients had a low $\mathrm{BMD}$, challenging the notion that osteoporosis is the causative factor in developing an insufficiency fracture, proposing that SIFK may be related to osteoarthritis instead. These findings are consistent with our case: our patient had an overall T-score of -0.7 with local osteopenia identified at the spine $(-1.2)$ and the neck of femur (-1.1). Instead of BMD, Nelson et al. ${ }^{7}$ argue that SIFK may be instead related to injuries associated with weight bearing abnormalities which occur in the setting of osteoarthritis, with their case series demonstrating a close association between SIFK and meniscal extrusion or injury. Our case did not support this notion-the SIFK to the lateral compartment was not accompanied by any concurrent degenerative changes. However, it should be noted that systemically decreased BMD may not necessarily reflect local BMD of the knee, which may play a role in the development of SIFK. We believe that the development of SIFK may be related to both a degree of local osteopenia, as well as degenerative processes, whether macro- or microscopic.

Our case presents the unusual occurrence of SIFK in the lateral compartment of the knee. SIFK/SONK lesions are more commonly described in the weight bearing medial articulation due to the associated repetitive loading ${ }^{4}$. These atypical lesions in the lateral compartment are even less easy to explain. Perhaps in cases of SIFK/SONK of the lateral femoral condyle, the inciting factor is the relative lower bone density in the lateral compartment compared to the medial compartment, a product of relative offloading of the lateral compartment, leading to disuse atrophy of the lateral femoral condyle as observed by Chiba et al. ${ }^{8)}$. This area of relative osteopenia would then be vulnerable to injury following repetitive loading. This may be the case in our patient who is a marathon athlete with mild valgus- the repetitive overload to a possibly mildly osteopenic lateral compartment may have resulted in the development of her insufficiency fracture.

Given the paucity of literature regarding SIFK, there are no standardised guidelines regarding its treatment. In general, nonoperative treatment regimes are centred around non- or partial weight bearing with an aim to optimise bone density via administration of bisphosphonate to prevent further subchondral collapse ${ }^{9)}$. Animal studies have demonstrated that bisphosphonates prevent early resorption of spontaneously necrotic bone, thus preventing structural failure and therefore progression to SIFK. Whilst this effect has been demonstrated in animal studies ${ }^{10,11}$, a randomized controlled trial demonstrated no significant difference between ibandronate treatment and placebo in $\mathrm{SONK}^{12}$. However, that there are no robust data regarding the use of these agents in SIF involving the knee, as well as the use of alternative bisphosphonate agents in the use of SONK and SIFK. The importance of physiotherapy should also not be ignored in the setting of SIFK, as it likely was a major contributor to our patients' rehabilitation.

Bisphosphonate therapy is not without its drawbacks, and has a variety of adverse effects ranging from short-term effects such as upper gastrointestinal upset to effects related to longer-term use such as osteonecrosis of the jaw, atrial fibrillation and suppression of bony turnover leading to atypical fractures ${ }^{13)}$.

Finally, if pain is non-responsive to conservative measures, or if mechanical symptoms arise due to loose body formation, surgical management may be warranted. Surgical interventions described in the literature include unicompartmental knee arthroplasty ${ }^{14)}$ as well as joint preserving procedures such as autologous osteochondral grafting or resurfacing procedures ${ }^{15)}$. However, like pharmacological management, there remains a lack of evidence for surgical treatment, which is somewhat unavoidable given the rare nature of this pathology.

In our case, our patient was managed conservatively with bisphosphonate therapy and was free of everyday symptoms at 18 months and at 30 months had regained full athletic function, reinforcing the notion that bisphosphonates are effective in the treatment of SIFK. The timeline of our patients' recovery is in keeping with those reported in the literature with regard to simi- 
lar injuries: a case series of subchondral impaction fractures of the lateral femoral condyle reports that healing continues up to 3 years postinjury ${ }^{16)}$.

Our case highlights a few pertinent points regarding the diagnosis and management of SIFK. The diagnosis of SIFK should be considered in any patient presenting with new-onset knee pain, particularly in active female patients with either local or systemically decreased BMD. Conservative treatment is first-line in the management of SIFK, although surgery may be indicated for non-remitting or mechanical symptoms. It should be noted that, as demonstrated by our case, SIFK can occur in the lateral compartment and therefore must always be considered as a potential cause for lateral knee pain.

\section{Conflict of Interest}

No potential conflict of interest relevant to this article was reported.

\section{References}

1. Wilmot AS, Ruutiainen AT, Bakhru PT, Schweitzer ME, Shabshin N. Subchondral insufficiency fracture of the knee: a recognizable associated soft tissue edema pattern and a similar distribution among men and women. Eur J Radiol. 2016;85:2096-103.

2. Ramnath RR, Kattapuram SV. MR appearance of SONK-like subchondral abnormalities in the adult knee: SONK redefined. Skeletal Radiol. 2004;33:575-81.

3. Kattapuram TM, Kattapuram SV. Spontaneous osteonecrosis of the knee. Eur J Radiol. 2008;67:42-8.

4. Yamamoto T, Bullough PG. Spontaneous osteonecrosis of the knee: the result of subchondral insufficiency fracture. J Bone Joint Surg Am. 2000;82:858-66.

5. Satku K, Kumar VP, Chong SM, Thambyah A. The natural history of spontaneous osteonecrosis of the medial tibial plateau. J Bone Joint Surg Br. 2003;85:983-8.

6. Akamatsu Y, Mitsugi N, Hayashi T, Kobayashi H, Saito T. Low bone mineral density is associated with the onset of spontaneous osteonecrosis of the knee. Acta Orthop. 2012; 83:249-55.
7. Nelson FR, Craig J, Francois H, Azuh O, Oyetakin-White P, King B. Subchondral insufficiency fractures and spontaneous osteonecrosis of the knee may not be related to osteoporosis. Arch Osteoporos. 2014;9:194.

8. Chiba K, Uetani M, Kido Y, Ito M, Okazaki N, Taguchi K, Shindo H. Osteoporotic changes of subchondral trabecular bone in osteoarthritis of the knee: a 3-T MRI study. Osteoporos Int. 2012;23:589-97.

9. Lai KA, Shen WJ, Yang CY, Shao CJ, Hsu JT, Lin RM. The use of alendronate to prevent early collapse of the femoral head in patients with nontraumatic osteonecrosis: a randomized clinical study. J Bone Joint Surg Am. 2005;87:21559.

10. Astrand J, Aspenberg P. Systemic alendronate prevents resorption of necrotic bone during revascularization: a bone chamber study in rats. BMC Musculoskelet Disord. 2002;3: 19.

11. Little DG, Peat RA, Mcevoy A, Williams PR, Smith EJ, Baldock PA. Zoledronic acid treatment results in retention of femoral head structure after traumatic osteonecrosis in young Wistar rats. J Bone Miner Res. 2003;18:2016-22.

12. Meier C, Kraenzlin C, Friederich NF, Wischer T, Grize L, Meier CR, Kraenzlin ME. Effect of ibandronate on spontaneous osteonecrosis of the knee: a randomized, doubleblind, placebo-controlled trial. Osteoporos Int. 2014;25:35966.

13. Kennel KA, Drake MT. Adverse effects of bisphosphonates: implications for osteoporosis management. Mayo Clin Proc. 2009;84:632-7.

14. Fujita S, Arai Y, Honjo K, Nakagawa S, Kubo T. A case of spontaneous osteonecrosis of the knee with early and simultaneous involvement of the medial femoral condyle and medial tibial plateau. Case Rep Orthop. 2016;2016:2574975.

15. Mistry H, Connock M, Pink J, Shyangdan D, Clar C, Royle P, Court R, Biant LC, Metcalfe A, Waugh N. Autologous chondrocyte implantation in the knee: systematic review and economic evaluation. Health Technol Assess. 2017;21:1-294.

16. Depasquale R, Fotiadou A, Kumar DS, Lalam R, Tins B, Tyrrell PN, Singh J, Cassar-Pullicino VN. Subchondral impaction fractures of the non-weight-bearing portion of the lateral femoral condyle. Skeletal Radiol. 2013;42:177-85. 